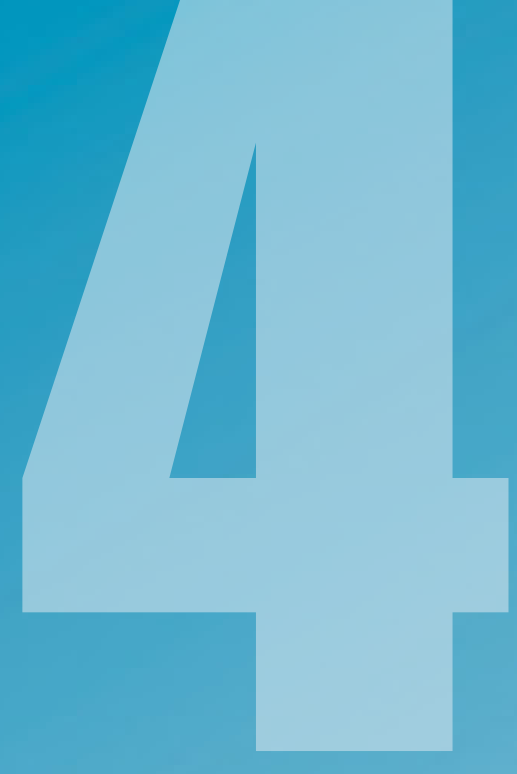

ÁMBITOS

REVISTA

INTERNACIONAL

DE COMUNICACIÓN

$N^{\circ} 46$

EDICIÓN OTOÑO

2019

ISSN: 1139-1979

E-ISSN: 1988-5733

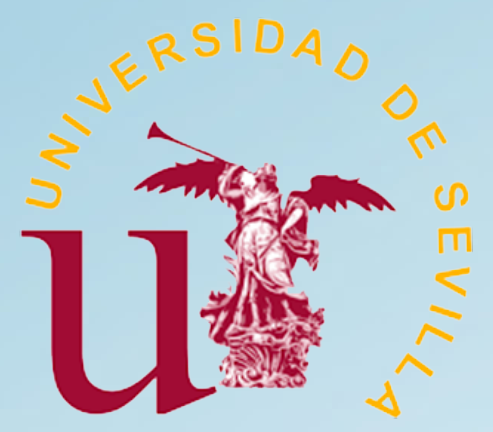




\section{ÍNDICE}

MONOGRAFICOS MONOGRAPHS

Presentación Monográfico. Comunicación emergente. Experiencias para el cambio social

Dra. Nereida López Vidales

Los formatos de televisión más consumidos por los jóvenes: telerrealidad y empoderamiento de la audiencia

Most consumed televisión formats by young people: real TV and the empowerment of the audience Nereida López Vidales, Leire Gómez Rubio, Elena Medina de la Viña

Nuevas herramientas, viejas costumbres El Contenido Generado por los Usuarios sobre el cambio climático en YouTube

New tools, old habits User Generated Content about climate change on YouTube

David Vicente Torrico

Tratamiento periodístico de personas LGTBIQ+ refugiadas: estudio de caso sobre Pride Barcelona 2018

Journalistic treatment of LGTBIQ+ refugees: case study on Pride Barcelona 2018

Hadriel Theodoro, Amparo Huertas Bailén

Tiempo Muerto, estudio de caso de un proyecto transmediático para la consecución de competencias universitarias

Tiempo Muerto, case study of a transmediatic project for the achievement of university competences Jose L. Carreño Villada, Miguel Ángel Díaz Monsalvo

Los universitarios millennials: uso de redes sociales y relación con las marcas

University Millennials: use of social media and engage with brands

Pedro Pablo Marín Dueñas, Esther Simancas González

Ali Ferzat: De la caricatura comunicativa en papel a la caricatura activista en los medios digitales

Ali Ferzat: From political cartoons in papers to political cartoons in digital media

Salud Adelaida Flores Borjabad

Direito à informação e literacia midiática: Reflexões sobre a questão do acesso

Right to information and media literacy: Reflections on the question of acce

Christiane Delmondes Versuti 
Eficacia de los influencers como recurso publicitario en la estrategia de los anunciantes locales Efficacy of influencers as an advertising resource in the strategy of local advertisers

Isabel Iniesta-Alemán

As redes sociais como ferramenta de marketing em instituições de ensino superior no Brasil Social networks as a marketing tool in higher education institutions in Brazil

Alcino Ricoy JR, Rogério Eduardo Rodrigues Bazi

Estudio sobre la conceptualización y el tratamiento informativo de la violencia de género en la prensa digital cubana

Study about the conceptualization and information treatment of gender violence in the Cuban digital press

Regla Ismaray Cabreja Piedra, Karina Escalona Peña

\section{ARTÍCULOS ARTICLES}

Uso de Twitter durante los debates electorales televisados en los comicios andaluces de 2018 The use of Twitter during the televised electoral debates in the 2018 Andalusian elections Julia Fontenla Pedreira, Erika Conde Vázquez, Carmen Máiz Bar

Quem averigua as notícias, os algoritmos ou jornalistas? A lógica crítica de C. S.

Peirce como processo de identificação de uma Fake News

Who checks the news, algorithms or journalists? The critical logic of C. S. Peirce as a

process for identifying a Fake News

Adelino de Castro Oliveira Simões Gala, Vania Baldi, Universidad de Aveiro

\section{RESEÑAS REVIEWS}

Nuevo ecosistema comunicativo digital: El consumidor

Juan Carlos Figuereo Benítez

Corpus toponímic de Beniarrés

Bianca Sánchez-Gutiérrez

The Future Computed. La inteligencia artificial y su papel en la sociedad y Pulsa actualizar. La aventura de redescubrir el alma de Microsoft y concebir un futuro mejor para todos 


\title{
Nuevas herramientas, viejas costumbres \\ El Contenido Generado por los Usuarios sobre el cambio climático en YouTube
}

\author{
New tools, old habits \\ User Generated Content about climate change on YouTube
}

David Vicente Torrico, Universidad de Valladolid, Plaza Campus Universitario, s/n, 47011, Valladolid

david.vicente.torrico@uva.es | Orcid: https://orcid.org/0000-0003-0379-6086

DOI: http://dx.doi.org/10.12795/Ambitos.2019.i46.03

\begin{abstract}
Resumen
El cambio climático es la principal amenaza medioambiental de nuestra era, no solo por sus dramáticas consecuencias, que año tras año van adquiriendo un mayor grado de certeza, sino también por su elevado nivel de contestación en el ámbito social e institucional. A ello contribuye una cobertura mediática duramente cuestionada por parte de los académicos, debido a la falta de contextualización, la atención prestada a voces escépticas, la presencia cíclica en la agenda y el tratamiento sensacionalista. En los últimos años, el desarrollo tecnológico ha potenciado la creación de contenidos por parte de los usuarios, en lo que se ha dado a conocer como CGU. YouTube suma 100 nuevas horas de contenidos cada minuto, y alberga una audiencia casi universal. Por estos motivos, consideramos relevante comprobar si esta nueva vía comunicativa ha permitido a los ciudadanos elaborar, o favorecer mediante su visualización, aquel tipo de información que demandan de los profesionales. Para ello proponemos un análisis de contenido sobre los vídeos creados por usuarios particulares, bajo la etiqueta de cambio climático, y situados entre los 50 que acumulan un mayor número de
\end{abstract}


visualizaciones. Los resultados obtenidos demuestran que los usuarios reproducen las mismas deficiencias detectadas en las coberturas profesionales, al priorizar las consecuencias sobre la contextualización, otorgar un amplio espacio a los escépticos y realizar un acercamiento basado en el dramatismo, tanto a nivel textual como visual.

\begin{abstract}
Climate change is the biggest environmental threat of our era, not only because of its dramatic consequences, which year after year are acquiring a greater degree of certainty, but also by their high level of social and institutional discussion. Media coverage is harshly questioned by academics due to the lack of contextualization, the attention given to sceptical voices, the cyclical presence on the agenda and the tabloid treatment contributes to this. In recent years, technological development has promoted the creation of content by users, also known as UGC. YouTube offers 100 new hours of content every minute, and has an almost universal audience. For these reasons, we consider to check if this platform has helped people to develop or to promote through visualization that kind of information demanded to journalists. In our study we share a content analysis of videos created by individual users, related to climate change, and located between the 50 who accumulate a higher number of displays. The results show that users play the same deficiencies identified in the coverage made by media, prioritizing the consequences on contextualization, giving an overrated space to skepticals and conducting an approach based on drama, both textual and visual.
\end{abstract}

Palabras clave: CGU, YouTube, Cambio climático, sensacionalismo, prosumidor.

Keywords: UGC, YouTube, Climate change, sensationalism, prosumer.

\title{
1. INTRODUCCIÓN
}

El cambio climático representa la principal amenaza medioambiental de nuestra era, no solo por sus consecuencias, que año tras año van adquiriendo un mayor grado de certeza, sino también por su elevado nivel de contestación en el ámbito social e institucional. Pese a los esfuerzos realizados durante los últimos años por parte de los colectivos científico, educativo y las organizaciones ecologistas, la mayor parte de los ciudadanos aún reconoce no sentirse debidamente informado en torno a este problema (Fundación Mapfre, 2009, 2011, 2013).

Las razones que podrían motivar este desconocimiento radican en la propia naturaleza del fenómeno, que se caracteriza por su lenta evolución y por impactar de manera más evidente sobre los territorios más alejados de las sociedades avanzadas. De este modo, la cobertura mediática realizada por los medios de comunicación se ha convertido en un agente clave a la hora de dar forma al imaginario social en torno al cambio climático, en 
una representación que ha sido tachada de sensacionalista y descontextualizada (Boykoff, 2010; León Anguiano y Erviti llundain, 2013), pero también de androcéntrica, eurocéntrica y excluyente (Lampis, 2013).

Por ello, desde el ámbito de la investigación proponemos analizar la utilización de otros modelos comunicativos, complementarios a los canales formativos e informativos habituales, que permitan a los ciudadanos acercarse a este problema desde un enfoque distinto, sin las limitaciones formales e ideológicas que marcan las rutinas de las redacciones periodísticas.

Mientras los medios de comunicación tradicionales priorizan los intereses empresariales en términos de audiencia por encima de la contextualización y la profundidad, fomentan la controversia frente al rigor científico y el debate frente al consenso, la Web 2.0 se presenta como el mejor escaparate para la creación y transmisión de la inteligencia colectiva, en un proceso de construcción democrática en el que la participación de los usuarios resulta fundamental para la elaboración y difusión del mensaje.

Los avances tecnológicos, con la mejora de la conexión de la red, la evolución de los dispositivos móviles y el auge de las redes sociales como máximos exponentes, han favorecido el desarrollo de un nuevo modelo de comunicación horizontal, en el que los roles de emisor y receptor se diluyen en favor de un ecosistema en el que los usuarios son los protagonistas, rompiendo así con la rigidez que caracteriza a los medios de comunicación tradicionales.

Este entorno, líquido y en constante cambio (Arroyo Almaraz y Baños González, 2013), invita a los internautas a crear, buscar, visionar, valorar y compartir contenidos, en definitiva, a diseñar su propia 'dieta informativa', liberándose con ello de la configuración de las agendas de los grupos periodísticos y de los intereses de los lobbies, y obteniendo de este modo una capacidad de decisión que les ha sido tradicionalmente negada en los círculos de poder, como son las grandes cumbres internacionales.

Pese a la oportunidad histórica que representa Internet para acceder directamente al conocimiento científico, ya sea a través de los propios informes o de los sitios web especializados y dependientes de este colectivo, la mayoría de los usuarios sigue acudiendo a los relatos audiovisuales para aprehender y comprender el mundo que les rodea (Nicholson-Cole, 2005), por lo que, a pesar de lo novedoso del entorno, reciben un mensaje mediado y, en definitiva, que ya ha sido interpretado por otros.

En este ecosistema digital, la plataforma de vídeo por excelencia es YouTube, que, aunque no es la única, sí es la que cuenta con un mayor volumen de visitas y la que ofrece un mayor número de contenidos, superando con ello a la suma de sus competidores más inmediatos (Pérez Rufi, 2013). 
Creada en 2005 por Jawed Karim, Steve Chen y Chad Hurley, YouTube se presenta como la televisión creada por y para el usuario, como una comunidad en constante actualización que fomenta la participación de sus miembros a través de sus múltiples posibilidades, a pesar de que, como comprobaremos en nuestro análisis, los índices de interacción son relativamente bajos. Tras su adquisición por parte de Google en 2006, el portal ha experimentado un crecimiento exponencial que le ha llevado a posicionarse como la segunda página web más visitada a nivel global según el ranking de Alexa ${ }^{1}$, solo por detrás de su matriz, el buscador de Google. Dada su popularidad, debemos considerar a YouTube como un poderoso agente de la industria mediática y, por lo tanto, como un actor relevante a la hora de conformar la opinión pública.

En YouTube conviven los perfiles profesionales, como las productoras, las agencias de publicidad o los medios de comunicación, que han aprovechado las características del portal para alargar la vida de sus contenidos y alcanzar a un público más amplio, con las obras que emergen de la cultura popular, en lo que se ha dado a conocer como el Contenido Generado por el Usuario (o User Generated Content en lengua inglesa). Este tipo de relatos, producidos de manera artesanal, han logrado obtener importantes cuotas de audiencia y generar pequeñas comunidades de suscriptores en torno a sus autores, que en algunos casos han pasado a dedicarse a la producción de contenidos audiovisuales como actividad profesional.

Según Núñez Gómez, García Guardia y Hermida Ayala (2012), los más jóvenes constituyen el público más activo en YouTube, mientras que el público de mayor edad solamente accede a esta plataforma para informarse acerca de temas específicos. Por esta razón, a nivel sociológico resulta de gran relevancia conocer qué tipo de vídeos son los mejor posicionados en torno a un asunto tan acuciante como lo es el deterioro medioambiental a escala planetaria.

En el ámbito académico, los contenidos alojados en este portal han servido como objeto de estudio en el campo de la imagen de los políticos, con el desarrollo de una narrativa que enfatiza su aspecto más humano (Vázquez Sande, 2016) desde un enfoque lúdico, alejado de su actividad profesional (Berrocal Gonzalo, Campos Domínguez y Redondo García, 2012). También hay ejemplos sobre la utilización de YouTube por parte de los movimientos sociales (Arévalo Salinas, 2014; Burgess y Green, 2018), las organizaciones no gubernamentales (Arroyo Almaraz, 2013), las empresas (Costa Sánchez, 2014) y las instituciones, con campañas de servicio público como la lucha contra la drogadicción (López Vidales y Gómez Rubio, 2015). Diferentes estudios, como el de Strangelove (2010), se acercan a la figura del prosumidor, es decir, del creador no profesional de contenidos para esta plataforma, mientras que otros como Keelan, Pavri García, Tomlinson y Wilson (2007) le confieren desde sus inicios la categoría de fuente de información, especialmente en el ámbito sanitario. 
Nos encontramos, por tanto, ante una fuente informativa de primer orden que, en los últimos años, ha irrumpido con fuerza en el ámbito de la investigación científica, debido a las audiencias millonarias que logra congregar a diario en su portal.

\section{OBJETIVOS E HIPÓTESIS}

El principal objetivo que persigue esta investigación es analizar la producción audiovisual generada por los usuarios del portal YouTube en torno a la representación del fenómeno del cambio climático.

Para ello, y a nivel específico, proponemos estudiar los 50 vídeos con mayor número de visualizaciones bajo la etiqueta de cambio climático, prestando una especial atención a los datos más representativos como el tipo de usuario, la duración, los recursos utilizados, el debate generado y la temática abordada en cada relato.

Nuestro punto de partida asume que los contenidos generados por los usuarios van a replicar en gran medida los defectos que estos mismos ciudadanos denuncian en la cobertura realizada por los medios de comunicación tradicionales.

De este modo, esperamos encontrar un tratamiento sensacionalista, con un gran peso de las voces escépticas, una limitada variedad de recursos audiovisuales, y un escaso impacto a nivel de debate generado, como, por otro lado, suele ser habitual en la red.

\section{METODOLOGÍA}

Para responder a estas cuestiones hemos realizado un análisis de contenido de los vídeos creados por los usuarios que aparecen entre los 50 vídeos más vistos sobre el cambio climático a fecha del 31 de diciembre del año 2018 (ver apéndice).

Con el objetivo de recopilar la mayor cantidad de información posible hemos optado por incorporar a nuestra herramienta de análisis los criterios específicos para el estudio de la recepción en YouTube utilizados por Arévalo Salinas $(2014,158)$ y Costa Sánchez $(2014,35)$. Sin embargo, y debido al cambio de la política informativa de la web, hemos tenido que prescindir de los datos de identificación de la audiencia, como la edad, la procedencia o el sexo. No obstante, como afirma Pérez Rufi (2013), este tipo de datos resulta arbitrario, ya que únicamente permite identificar a aquellos usuarios registrados, sin existir ningún tipo de control sobre la veracidad de los mismos.

En el apartado temático (Vicente Torrico, 2017) apostamos por el estudio de 8 categorías temáticas que agrupan las variables más representativas del cambio climático: 
- Causas humanas: El cambio climático de origen antropogénico se caracteriza por las emisiones de gases de efecto invernadero, pero también por el acceso y uso de los recursos disponibles y de la superficie terrestre.

- Causas naturales: Los agentes naturales, tales como los cambios en la superficie solar, la actividad volcánica, las variaciones en el campo magnético terrestre, el impacto de meteoritos y las alteraciones de la órbita terrestre, representan un factor de variabilidad climática.

- Consecuencias sobre la superficie helada: Los hielos se ven afectados mediante la reducción del efecto albedo, el deshielo de los glaciares y de los polos.

- Consecuencias sobre la superficie oceánica: Los océanos ven alteradas sus corrientes, la composición química del agua, pero también experimentan la elevación del nivel del mar y favorecen la aparición de fenómenos extremos.

- Consecuencias sobre la superficie terrestre: El cambio climático produce cambios en los ecosistemas, conflictos y movimientos migratorios, escasez de recursos, cambios en el régimen de lluvias y en las temperaturas, pérdidas de rendimiento de los cultivos y amenaza los sistemas de salud pública.

- Soluciones mitigadoras: Entre las propuestas para erradicar el cambio climático se presentan las energías renovables, la geoingeniería, los mercados de emisiones, la reducción de emisiones, la reforestación y los sumideros de carbono.

- Soluciones preventivas: Las medidas que tratan de anticiparse a los impactos del cambio climático ya esperados consisten en alcanzar acuerdos políticos, instaurar sistemas de alerta temprana, de denuncia, programas de educación ambiental, invertir en infraestructuras resistentes a fenómenos y condiciones extremas, la movilización social y la protección de espacios y especies.

- Soluciones reactivas: Las iniciativas para enfrentarse a las consecuencias del cambio climático una vez que se han producido se centran en mejorar la eficiencia del sistema productivo, el diseño de planes de evacuación, el fomento del reciclaje y la recuperación de los ecosistemas que ya se han visto dañados.

Por tanto, los 50 vídeos sobre el cambio climático con un mayor número de reproducciones en la página de YouTube han sido analizados a partir de esta plantilla: 


\section{Tabla 1}

Instrumento de análisis para los vídeos seleccionados

\begin{tabular}{|c|c|c|c|}
\hline Orden & Título del vídeo & & \\
\hline 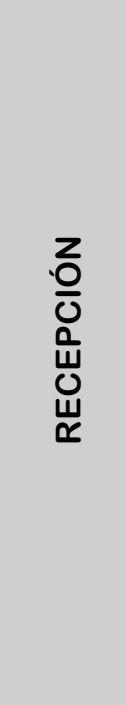 & $\begin{array}{l}\text { Autor } \\
\text { Fecha de subida } \\
\text { Duración } \\
\text { Número reproducciones } \\
\text { Número comentarios } \\
\text { Me gusta / No me gusta }\end{array}$ & 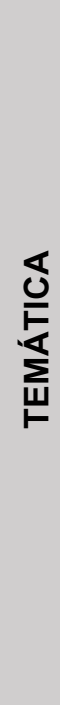 & $\begin{array}{l}\text { Causas humanas } \\
\text { Causas naturales } \\
\text { Consecuencias hielos } \\
\text { Consecuencias océanos } \\
\text { Consecuencias terrestres } \\
\text { Soluciones mitigación } \\
\text { Soluciones prevención } \\
\text { Soluciones reacción }\end{array}$ \\
\hline
\end{tabular}

Fuente: Elaboración propia

Dado que nuestro interés se centra en los contenidos generados por los usuarios, en esta comunicación hemos seleccionado los vídeos que pertenecen a cuentas y canales no profesionales, por lo que quedan excluidos del análisis los relatos que no son originales y todas aquellas cuentas asociadas a empresas, asociaciones, productoras audiovisuales, medios de comunicación e instituciones.

En consecuencia, de los 50 vídeos seleccionados inicialmente, nuestro material de trabajo para analizar la representación del cambio climático realizada por los internautas ha quedado configurado por un total de 23 creaciones.

\section{RESULTADOS VINCULADOS A LA CREACIÓN Y RECEPCIÓN}

Las estadísticas obtenidas demuestran que el relato sobre el cambio climático en YouTube aparece dominado, en términos cuantitativos, por el autor no profesional, con un total de 34 resultados. De estos, 11 se corresponden con contenidos con derechos de autor, es decir, que no pertenecen al usuario que los comparte.

Los medios de comunicación obtienen el mejor resultado entre las cuentas profesionales, con 5 vídeos, y han logrado situar a 2 de ellos como los más vistos. Se 
trata de breves relatos que tienen a La familia Telerín como protagonistas, y que suman en conjunto más de 21 millones de reproducciones. Las ONGs y las instituciones consiguen colocar 3 títulos entre los 50 más vistos, mientras que las empresas, en concreto Acciona, y las productoras independientes aportan únicamente 2 al listado.
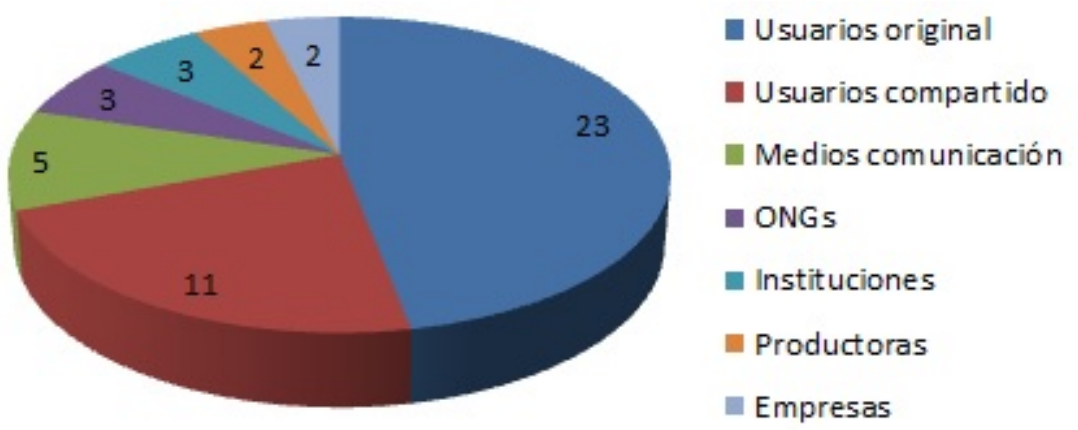

Figura 1. Tipología de usuarios en los vídeos seleccionados Fuente: Elaboración propia

Si profundizamos en las cuentas de los usuarios, comprobamos que hay dos perfiles particulares que contribuyen a la muestra con 3 vídeos cada uno. Se trata del canal El Robot de Platón, cuyo contenido está dedicado a la divulgación científica, y de la cuenta Quantum Fracture, en la que José Luis Crespo presenta vídeos didácticos sobre ciencia $y$, al menos en el caso que nos ocupa, cuenta con el patrocinio de Iberdrola.

\section{Tabla 2}

Fecha de subida y reproducciones acumuladas de los vídeos (datos en miles)

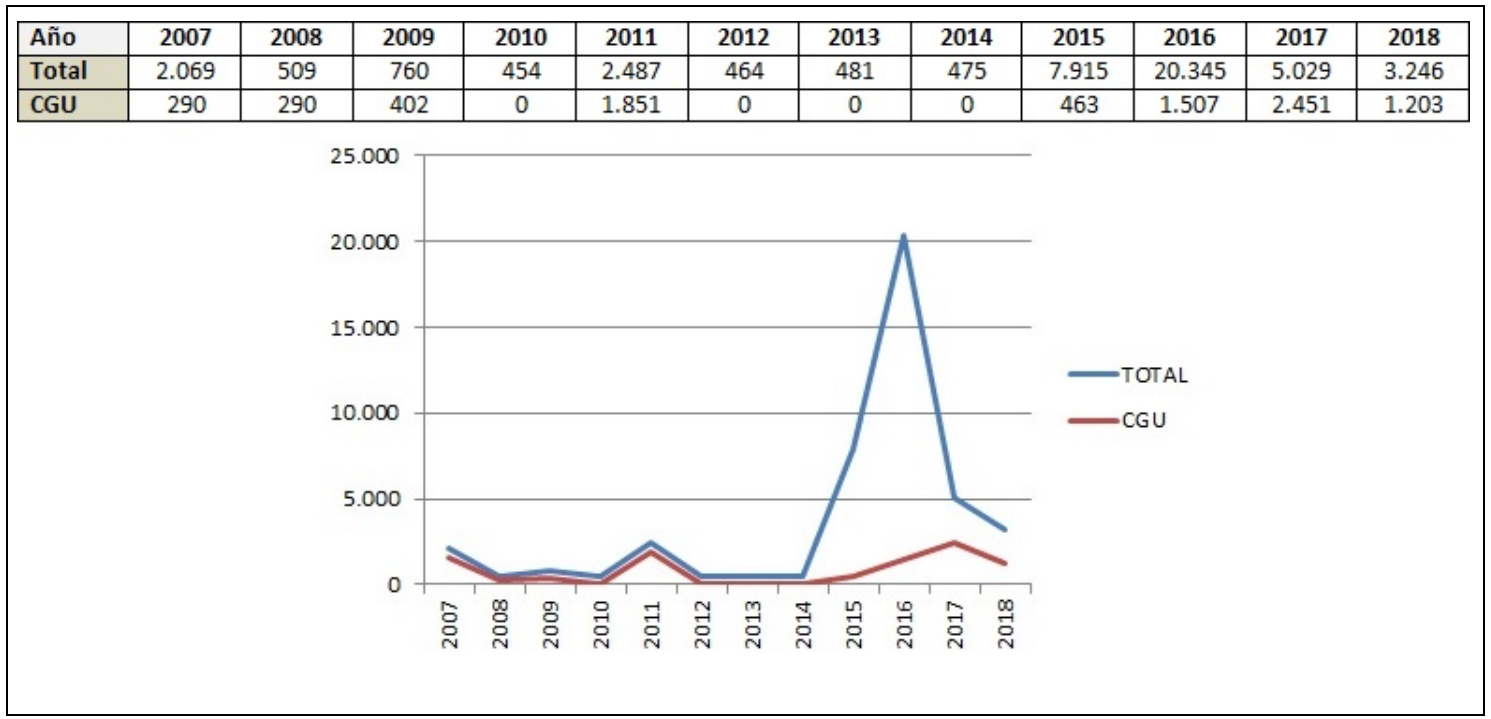

Fuente: Elaboración propia 
Si atendemos a las fechas de subida, comprobamos que es un asunto de absoluta actualidad, ya que el $80 \%$ de las visualizaciones se concentra en vídeos producidos durante los últimos 4 años. Sin embargo, en el caso analizado, este impacto se reduce hasta el $60 \%$. La popularidad de los vídeos de La Familia Telerín, que multiplican en reproducciones a los siguientes relatos, marcan la diferencia en este apartado.

Respecto a la repercusión social, los resultados demuestran que el contenido generado por los usuarios despierta un notable interés entre la comunidad, ya que los 5 primeros vídeos acumulan más de 4.000 comentarios. Destaca el usuario Quantum Fracture, que, merced a una cuidada presentación y a la interacción con el público, logra situar 2 de sus producciones al frente del listado, como muestra la siguiente tabla.

Tabla 3

Vídeos que acumulan un mayor número de comentarios

\begin{tabular}{|l|l|l|l|}
\hline & Orden Visualizaciones & Usuario & Comentarios \\
\cline { 2 - 4 } & 18 & QuantumFracture (CGU) & 4.576 \\
\cline { 2 - 4 } & 15 & QuantumFracture (CGU) & 4.572 \\
\hline 14 & Draw Club (CGU) & 4.481 \\
\cline { 2 - 4 } & 4 & Colthrone (CGU) & 4.163 \\
\cline { 2 - 4 } & 6 & EnigmaCinco (CGU) & 3.725 \\
\hline
\end{tabular}

Fuente: Elaboración propia

Para situar este dato en su contexto, debemos señalar que los 50 vídeos más vistos en YouTube sobre el cambio climático suman un total de 43.661 comentarios, lo que arroja un promedio de casi 900 para cada vídeo. En el caso del contenido analizado la cifra asciende a los 37.451 , y la media aumenta hasta los 1.628 resultados. En este sentido parece que la tipología del autor supone, por tanto, un factor a tener en cuenta.

Otra de las cualidades que parece haber influido de manera notable en el número de comentarios generados es la propia temática abordada en el vídeo. Sin profundizar en las variables, que lo haremos más adelante, hemos podido detectar que los vídeos que presentan planteamientos escépticos, es decir, aquellos que cuestiona el origen antropogénico del cambio climático, suscitan una gran participación entre los espectadores, del mismo modo que las referencias a las consecuencias del cambio climático. 


\section{Tabla 4}

Vídeos que acumulan un mayor número de votos positivos

\begin{tabular}{|l|l|l|l|}
\hline Orden Visualizaciones & Usuario & Votos positivos \\
\cline { 2 - 4 } & 15 & QuantumFracture (CGU) & 52.000 \\
\hline 17 & QuantumFracture (CGU) & 51.000 \\
\hline 7 & QuantumFracture (CGU) & 37.000 \\
\hline 18 & El Robot de Platón (CGU) & 37.000 \\
\cline { 2 - 4 } & 3 & Roggy TM 73 (CGU) & 32.000 \\
\hline
\end{tabular}

Fuente: Elaboración propia

En cuanto a las puntuaciones, las creaciones de los usuarios reciben las mejores valoraciones, ya que el primer vídeo profesional aparece en el séptimo puesto, de la mano de Acciona. De este modo, la clasificación global aparece dominada por 6 relatos amateur que suman más de 30.000 votos positivos cada uno. Al frente se encuentran los vídeos de Quantum Fracture, que alcanzan en 2 de ellos la cifra de 50.000 likes.

En el lado negativo, comprobamos que el número de reproducciones supone un factor determinante, ya que los 5 vídeos con peor valoración se encuentran entre las 6 primeras posiciones en cuanto a número de visualizaciones. En este sentido, tras los relatos sobre La Familia Telerín, de Clan TV, son vídeos creados por usuarios los que acumulan las peores valoraciones. Así, el relato en clave religiosa de Colthrone y el amplio abanico de consecuencias que muestran Roggy TM 73 y EnigmaCinco han sido las creaciones realizadas por aficionados con una peor acogida entre el público.

Tabla 5

Vídeos que acumulan un mayor número de votos negativos

\begin{tabular}{|l|l|l|l|}
\hline & Orden Visualizaciones & Usuario & Votos negativos \\
\cline { 2 - 4 } & 1 & Cleo \& Cuquín - Familia Telerín (TV) & 12.000 \\
\cline { 2 - 4 } & 2 & Cleo \& Cuquín - Familia Telerín (TV) & 3.600 \\
\hline 4 & Roggy TM 73 (CGU) & 3.500 \\
\hline \multirow{2}{*}{3} & Colthrone (CGU) & 2.600 \\
\cline { 2 - 4 } & 6 & EnigmaCinco (CGU) & 1.400 \\
\hline
\end{tabular}

Fuente: Elaboración propia 
En último lugar, y para cerrar el apartado de las estadísticas que ofrece YouTube, analizamos la duración de los vídeos seleccionados. De este modo, los 50 relatos sobre el cambio climático con un mayor número de reproducciones suman un total de 670 minutos, lo que representa un tiempo promedio de algo más de 13 minutos por vídeo. En el caso de los contenidos generados por los usuarios, la duración media se sitúa por debajo de los 10 minutos.

Esta circunstancia se debe a que entre los vídeos asociados a las cuentas profesionales y los contenidos con derechos de autor compartidos por otros usuarios existen varios documentales, cuya duración se acerca a los 90 minutos. Así, entre los relatos cinematográficos compartidos encontramos el documental Home (2009), dirigido por Yann Arthus Bertrand, y la cinta de contenidos escépticos La gran estafa del calentamiento global (2007), a cargo de Martin Durkin. En el lado de los contenidos televisivos podemos hablar de la serie Aire: Cambio climático (2011), del canal argentino Encuentro, y del documental Planeta Futuro (2009), creado por la BBC británica.

Si nos centramos en los contenidos generados por los usuarios, destacan 2 vídeos por superar ampliamente el tiempo medio de este tipo de relatos. Así, los consultorios de Misterios al descubierto y Mundo desconocido alcanzan una duración de 54 y 23 minutos respectivamente, si bien se basan únicamente en la exposición de sus opiniones ante la webcam.

\section{Tabla 6}

Vídeos seleccionados con mayor duración

\begin{tabular}{|l|l|l|l|}
\hline & Orden Visualizaciones & Usuario & Duración \\
\cline { 2 - 4 } & 21 & Isabel Ramírez (comp.) & $1: 33: 17$ \\
\cline { 2 - 4 } & 8 & VHS 4000 (comp.) & $1: 28: 19$ \\
\cline { 2 - 4 } & 20 & Eduzeitgeist (comp.) & $1: 15: 56$ \\
\cline { 2 - 4 } & 35 & Misterios al Descubierto (comp.) & $54: 06$ \\
\cline { 2 - 4 } & 50 & Fernando Pablo Mori (comp.) & $26: 02$ \\
\hline
\end{tabular}

Fuente: Elaboración propia

\section{RESULTADOS VINCULADOS A LA TEMÁTICA ANALIZADA}

Además del estudio de la repercusión en términos numéricos de los contenidos generados por los usuarios de YouTube en torno al cambio climático, el objetivo de esta comunicación reside en comprobar qué tipo de representación realizan acerca de este fenómeno. 
De este modo, y para adentrarnos en el estudio de las variables temáticas utilizadas, partimos de la constatación de la presencia de un total de 6 vídeos vinculados a la corriente de opinión escéptica, lo que supone más de una cuarta parte de la muestra. Se trata de relatos basados en la opinión de los propios usuarios, en los que, con un primer plano y sus propios conocimientos como únicos recursos disponibles, cuestionan el consenso científico en torno al origen, las consecuencias y los posibles tratamientos a realizar para combatir el cambio climático.

Respecto al origen del cambio climático, que aparece en 47 ocasiones, el $60 \%$ de las menciones están vinculadas a las causas antropogénicas, con las emisiones de gases de efecto invernadero como el principal exponente de este problema. El $40 \%$ restante de los comentarios aluden a los agentes naturales, si bien en más de la mitad de las ocasiones lo hacen para criticar los planteamientos escépticos, por lo que la incidencia del hombre sobre el clima se hace aún más palpable en nuestro material de análisis.

Tabla 7

Vídeos seleccionados con mayor duración

\begin{tabular}{|l|l|c|c|}
\hline Categoría & Variable & Positivo & Negativo \\
\hline \multirow{4}{*}{ Causas humanas (29) } & Emisiones GEI (18) & 16 & 2 \\
\cline { 2 - 4 } & Acceso y uso recursos (6) & 6 & 0 \\
\cline { 2 - 4 } & Acceso y uso suelo (5) & 5 & 0 \\
\hline \multirow{5}{*}{ Causas naturales (18) } & Actividad solar (6) & 3 & 3 \\
\cline { 2 - 4 } & Actividad volcánica (6) & 2 & 4 \\
\cline { 2 - 4 } & Campo magnético (2) & 1 & 1 \\
\cline { 2 - 4 } & Impacto meteoritos (1) & 0 & 1 \\
\cline { 2 - 4 } & Órbita terrestre (3) & 1 & 2 \\
\hline
\end{tabular}

Fuente: Elaboración propia

Las consecuencias del cambio climático representan la categoría más frecuente, con un total de 89 alusiones. De entre las superficies afectadas, la terrestre es la que más resultados obtiene, con cerca del $60 \%$, mientras que la superficie oceánica y la helada quedan a una notable distancia, con un $23 \%$ y un $17 \%$ respectivamente.

Si analizamos las variables de manera individual, los cambios de temperatura son el tema más relevante para los autores de los vídeos, al aparecer en un total de 16 vídeos, con 14 menciones al incremento de temperatura y 6 al descenso térmico. 
En un segundo peldaño se encuentran las alteraciones en los patrones de lluvia, que aparecen en 14 ocasiones. Los discursos otorgan la misma importancia tanto a las inundaciones como a las sequías, con 7 intervenciones en cada caso.

El deterioro de los ecosistemas es el tercer efecto del cambio climático más recurrente, con 10 apariciones, mientras que el incremento del nivel del mar, la formación de fenómenos extremos y el deshielo de los polos suman 9 resultados.

\section{Tabla 8}

Resultados obtenidos en el estudio de las consecuencias del cambio climático

\begin{tabular}{|l|l|c|c|}
\hline Categoría & Variable & Positivo & Negativo \\
\hline \multirow{4}{*}{ Causas humanas (29) } & Emisiones GEI (18) & 16 & 2 \\
\cline { 2 - 4 } & Acceso y uso recursos (6) & 6 & 0 \\
\cline { 2 - 4 } & Acceso y uso suelo (5) & 5 & 0 \\
\hline \multirow{5}{*}{ Causas naturales (18) } & Actividad solar (6) & 3 & 3 \\
\cline { 2 - 4 } & Actividad volcánica (6) & 2 & 4 \\
\cline { 2 - 4 } & Campo magnético (2) & 1 & 1 \\
\cline { 2 - 4 } & Impacto meteoritos (1) & 0 & 1 \\
\cline { 2 - 4 } & Órbita terrestre (3) & 1 & 2 \\
\hline
\end{tabular}

Fuente: Elaboración propia

Las soluciones al cambio climático constituyen la categoría temática con menor incidencia en la muestra analizada, ya que aparecen en 43 ocasiones. El relato generado pone el foco sobre las medidas preventivas, que representan casi la mitad de los resultados, por delante de los proyectos de mitigación y de las propuestas encaminadas a la reacción ante los impactos esperados, que aportan el $32 \%$ y el $18 \%$ de los casos.

A nivel particular, la movilización social es la solución preferida por los autores, que apuestan por ella en 7 ocasiones. Con idéntica presencia en el estudio se encuentran las energías renovables, si bien en uno de los casos el discurso es negativo, al hablar del desvío de fondos públicos hacia lobbies verdes. El reciclaje ocupa el tercer lugar, con 4 intervenciones, seguido con 3 por la protección del entorno, la mejora de la eficiencia energética y la reducción de emisiones de gases de efecto invernadero.

En el lado negativo, los internautas se muestran contrarios hacia los acuerdos políticos, ya que de las 5 veces en las que se mencionan, 4 son para criticar su incapacidad como gestores. Lo mismo sucede con la inversión económica en infraestructuras resistentes a los impactos del cambio climático, ya que son tachadas de innecesarias y fraudulentas 
en 3 ocasiones. Por último, en una ocasión se ironiza acerca de la posibilidad de evacuar a la población, toda vez que el planeta se haya vuelto inhabitable.

\section{Tabla 9}

Resultados obtenidos en el estudio de las consecuencias del cambio climático

\begin{tabular}{|c|c|c|c|}
\hline Categoría & Variable & Positivo & Negativo \\
\hline \multirow{6}{*}{ Soluciones mitigación (14) } & Renovables (7) & 6 & 1 \\
\hline & Geoingeniería (1) & 1 & 0 \\
\hline & Mercado GEI (0) & 0 & 0 \\
\hline & Reducción GEI (3) & 3 & 0 \\
\hline & Reforestación (2) & 2 & 0 \\
\hline & Sumideros carbono (1) & 1 & 0 \\
\hline \multirow{7}{*}{ Soluciones prevención (21) } & Acuerdos políticos (5) & 1 & 4 \\
\hline & Alerta temprana (0) & 0 & 0 \\
\hline & Denuncia (0) & 0 & 0 \\
\hline & Educación ambiental (2) & 2 & 0 \\
\hline & Inversión infraestructuras (4) & 1 & 3 \\
\hline & Movilización social (7) & 7 & 0 \\
\hline & Protección (3) & 3 & 0 \\
\hline \multirow{4}{*}{ Soluciones reacción (8) } & Eficiencia (3) & 3 & 0 \\
\hline & Evacuación (1) & 0 & 1 \\
\hline & Reciclaje (4) & 4 & 0 \\
\hline & Recuperación (0) & 0 & 0 \\
\hline
\end{tabular}

Fuente: Elaboración propia

\section{CONCLUSIONES Y DISCUSIÓN}

Los resultados obtenidos al analizar la muestra de los 50 vídeos con mayor número de visualizaciones en torno a la representación del cambio climático en YouTube nos ha permitido confirmar nuestro punto de partida, en el que señalábamos que, pese a tratarse de un nuevo medio audiovisual, con el espectador como protagonista de un entorno libre y democrático, el mensaje creado por los usuarios no distaría demasiado de la representación del fenómeno que realizan los medios de comunicación tradicionales. Por ello, y a pesar de la notable repercusión que han logrado generar los vídeos en término de número de reproducciones, podemos concluir que su visualización no contribuye a superar la situación del desconocimiento existente en torno a la problemática del cambio climático entre la sociedad. 
En lo referido a la producción del material audiovisual, los usuarios constituyen la primera fuente documental según la muestra analizada, ya que son los responsables de casi la mitad de los vídeos sobre el cambio climático, situándose muy por delante de la industria profesional. Por lo tanto, y a diferencia de lo señalado en su proyecto por Pérez Rufi $(2013,45)$, el contenido creado por los internautas juega un papel fundamental en la representación en línea del tema abordado.

El carácter no profesional de estos vídeos, pese a la calidad que presentan algunos de ellos, provoca que en la mayoría de las ocasiones se impongan las ideas sobre las formas, con la utilización mayoritaria de bustos parlantes e imágenes de recurso frente a la producción de material propio y original, en una tendencia que ya fue detectada en su estudio por Arroyo Almaraz y Baños González $(2013,621)$.

A pesar de las limitaciones técnicas antes indicadas, el abordaje realizado por los internautas no está exento de unos elevados índices de sensacionalismo y dramatismo, ya que casi la mitad de las menciones al cambio climático se enfocan desde la perspectiva de las consecuencias. Esta representación, en la que se enfatizan los efectos provocados por la amenaza, dificulta la correcta contextualización del problema medioambiental, ya que el espacio dedicado a las causas y, especialmente, a las soluciones es reducido.

La sobreexposición a los efectos del cambio climático, especialmente en aquellas variables que inciden directamente sobre la población y sus condiciones de vida, es una constante en la representación mediática del problema, tanto en España (Erviti Ilundain y De Lara González, 2012; Heras Hernández, 2013) como en el extranjero (Carvalho, 2012; Djerf-Pierre, 2012).

Por el contrario, las consecuencias a largo plazo, que son aquellas que abordan las afecciones a la salud, la pérdida de rendimiento de los cultivos y la destrucción de entornos habitables, reciben una atención menor por parte de los creadores profesionales, como ya indicaran en sus estudios los equipos de Maybach (2010) y Myers (2012), en una dinámica que también se reproduce en el contenido generado por los usuarios que hemos analizado.

Esta situación de invisibilidad se manifiesta igualmente en lo referido a las soluciones dirigidas a mitigar el problema, cuya presencia en los relatos estudiados es inferior a la tercera parte de las medidas propuestas, una circunstancia que ya fue denunciada por Fernández Reyes en su estudio sobre la prensa española (2014). No obstante, esta circunstancia puede resultar positiva a la hora de involucrar a la audiencia, ya que, como señala Heras González (2013), los vídeos muestran opciones de actuación al alcance de cualquier ciudadano. 
En cuanto a la recepción de los vídeos, hemos comprobado que los relatos generados por los usuarios en torno al cambio climático han generado, en términos globales, un nivel de impacto considerablemente reducido en cuanto a interacciones.

De este modo, tan solo el $0,25 \%$ de la audiencia otorgó un voto positivo al contenido, aunque esta cifra multiplica en más de 27 veces el número de votos negativos, que representa el $0,09 \%$ de visionados. El número de comentarios también demuestra una cierta pasividad por parte de la comunidad, al alcanzar el 2,5\%.

Bien es cierto que, según las conclusiones de Gallardo y Jorge (2010), el promedio de comentarios en la plataforma de Youtube se sitúa en torno al $0,15 \%$ en el caso de los vídeos más vistos. Sin embargo, y considerando la trascendencia de este problema, resulta una oportunidad perdida para una sociedad moderna que reclama su espacio en la toma de decisiones en torno a un problema de repercusiones globales.

\section{Notas}

${ }^{1}$ El índice Alexa aparece referenciado en la web: https://www.alexa.com/topsites

\section{Referencias}

Arévalo Salinas, A.I. (2014). El movimiento social 15-M de España y la promoción de la protesta a través de sus vídeos en YouTube. Historia y Comunicación Social, 19, 153-163.

Arroyo Almaraz, I. y Baños González, M. (2013). La eficacia de la comunicación de las organizaciones del Tercer Sector en los vídeos emitidos a través de YouTube. Historia y Comunicación Social, 18, 615-626.

Berrocal Gonzalo, S., Campos Domínguez, E. y Redondo García, M. (2012). Comunicación política en Internet: La tendencia al "infoentretenimiento" político en YouTube. Estudios sobre el mensaje periodístico, 18(2), 643-659.

Boykoff, M.T. (2010). Indian media representations of climate change in a threatened journalistic ecosystem. Climatic Change, 99, 17-25.

Burgess, J. y Green, J. (2018). YouTube: Online video and participatory culture. Oxford, Reino Unido: John Wiley \& Sons.

Carvalho, A. (2012). Cambio climático, medios de comunicación y la paradoja del conocimiento y la inacción. En J.L. Piñuel Raigada et al. (Eds.) Comunicación, controversias e incertidumbres frente al consenso científico acerca del cambio climático. La Laguna, España: Cuadernos Artesanos La Latina.

Costa Sánchez, C. (2014). Audiovisual y Web 2.0. Empleo de YouTube por las empresas españolas con mejor reputación. Chasqui. Revista Latinoamericana de Comunicación, 126, 31-41. 
Djerf-Pierre, M. (2012). When attention drives attention: Issue dynamics in environmental news reporting over five decades. European Journal of Communication, 27(3), 291-304.

Erviti Ilundain, M.C. y de Lara González, A. (2012). Las imágenes televisivas del cambio climático. Estudio de la cobertura de la Cumbre de Durban en los informativos españoles. En Comunicación: revista Internacional de Comunicación Audiovisual, Publicidad y Estudios Culturales, 10, pp. 1467-1482.

Fernández Reyes, R. (2014). Infoxicación en la comunicación del cambio climático. Mitigación y adaptación. Ámbitos. Revista Internacional de Comunicación 26, 1-12. doi: 10.12795/Ambitos.2019.i46.03

Fundación Mapfre (2009). La sociedad ante el cambio climático. Conocimientos, valoraciones y comportamientos en la población española. Ferrol, España: Aldine.

Fundación Mapfre (2011). La sociedad ante el cambio climático. Conocimientos, valoraciones y comportamientos en la población española. Ferrol, España: Aldine.

Fundación Mapfre (2013). La respuesta de la sociedad española ante el cambio climático. Ferrol, España: Aldine.

Gallardo, J. y Jorge, A. (2010). La baja interacción del espectador de vídeos en Internet: caso Youtube España. Revista Latina de Comunicación Social, 65, 421-435.

Heras Hernández, F. (2013). Una de acción: el tratamiento mediático de las soluciones al cambio climático. Razón y Palabra, 18(84), 232-244.

Keelan, J., Pavri García, V., Tomlinson, G. y Wilson, K. (2007). YouTube as a source of information on immunization: a content analysis. JAMA, 298(21), 2482-2484.

Lampis, A. (2013). La adaptación al cambio climático: El reto de las dobles agendas. En J.C. Postigo (ed.) Cambio climático, movimientos sociales y políticas públicas: una vinculación necesaria (pp.29-50). Santiago de Chile, Chile: Clacso.

León Anguiano, B. y Erviti, M.C. (2015). Science in pictures: Visual representation of climate change in Spain's television news. Public Understanding of Science, 24(2), 183-199.

López Vidales, N. y Gómez Rubio, L. (2015). Análisis y proyección de los contenidos audiovisuales sobre jóvenes y drogas en YouTube. Estudios sobre el mensaje periodístico, 21(2), 863-881.

Nicholson-Cole, S.A. (2005). Representing climate change futures: a critique on the use of images for visual communication. Computers, environment and urban systems, 29(3), 255-273.

Pérez Rufi, J.P. (2013). La actualidad en YouTube: claves de los vídeos más vistos durante un mes. Global Media Journal México, 9(17), 44-62.

Strangelove, M. (2010). Watching YouTube: Extraordinary videos by ordinary people. Toronto, Canadá: University of Toronto Press. 
Vázquez Sande, P. (2016). Storytelling personal en política a través de Youtube. Comunicación y Hombre, 12, 41-55.

Vicente Torrico, D. (2017). Diseño metodológico para el estudio de la representación cinematográfica del fenómeno del cambio climático. Actas del IV Congreso Nacional de Metodología de la Investigación en Comunicación. Castellón, España. 


\section{APÉNDICE}

Vídeos sobre cambio climático creados por los usuarios y entre la lista de los $\mathbf{5 0}$ más vistos (a 31 de diciembre de 2018)

\begin{tabular}{|c|c|c|c|}
\hline Orden & Autor & Título & Fecha \\
\hline 3 & Roggy TM 73 & $\begin{array}{l}\text { Top } 7 \text { cambios climáticos más aterradores del } \\
\text { mundo }\end{array}$ & $27 / 09 / 2016$ \\
\hline 4 & Colthrone & No... no es cambio climático & 08/09/2017 \\
\hline 5 & Criss++ & $\begin{array}{l}\text { Calentamiento global - Salvemos al globo del } \\
\text { cambio climático }\end{array}$ & $18 / 09 / 2011$ \\
\hline 6 & EnigmaCinco & $\begin{array}{l}\text { [Simulación] Así quedaría tu país tras el cambio } \\
\text { climático }\end{array}$ & 09/05/2017 \\
\hline 7 & El Robot de Platón & El cambio climático explicado & $20 / 09 / 2016$ \\
\hline 9 & Edu0404Buffon & Cambio climático - consecuencias - & $14 / 05 / 2007$ \\
\hline 11 & CuriosaMente & ¿Es real el cambio climático? & $15 / 05 / 2016$ \\
\hline 13 & Eraklito & Cambio climático e indolencia & $30 / 01 / 2007$ \\
\hline 14 & Draw Club & ¿Qué nos ocurrirá con el cambio climático? & 09/07/2017 \\
\hline 15 & QuantumFracture & $\begin{array}{l}\text { El cambio climático es culpa nuestra y puedo } \\
\text { convencerte }\end{array}$ & $04 / 12 / 2018$ \\
\hline 16 & Simon Wilches & $\begin{array}{l}\text { Qué es cambio climático? What's climate } \\
\text { change? }\end{array}$ & $08 / 12 / 2009$ \\
\hline 17 & QuantumFracture & $\begin{array}{l}\text { Lo que Trump (y tú) deberíais saber sobre el } \\
\text { cambio climático }\end{array}$ & $25 / 10 / 2018$ \\
\hline 18 & QuantumFracture & $\begin{array}{l}\text { Respondo comentarios de negacionistas del } \\
\text { cambio climático }\end{array}$ & $10 / 12 / 2018$ \\
\hline 24 & $\begin{array}{l}\text { Mundo } \\
\text { desconocido }\end{array}$ & Un negocio llamado "cambio climático" & $25 / 06 / 2015$ \\
\hline 25 & VM Granmisterio & $\begin{array}{l}\text { Los Rockefeller están preparando algo muy } \\
\text { siniestro }\end{array}$ & $14 / 02 / 2017$ \\
\hline 27 & El Robot de Platón & Mitos climáticos & 03/07/2017 \\
\hline
\end{tabular}

Ámbitos. Revista Internacional de Comunicación | ISSN: 1139-1979 | E-ISSN: 1988-5733, №. 46. (2019) 


\begin{tabular}{|c|l|l|l|}
\hline Orden & Autor & Título & Fecha \\
\hline 28 & Chemtrailsno & $\begin{array}{l}\text { ¿Por qué no llueve? La mentira sobre el cambio } \\
\text { climático. Parte I }\end{array}$ & $04 / 02 / 2008$ \\
\hline 32 & El Robot de Platón & Cómo retrasar el cambio climático & $21 / 03 / 2017$ \\
\hline 33 & Antonio Pareja & Cambio climático, el documental & $02 / 10 / 2011$ \\
\hline 35 & $\begin{array}{l}\text { Misterios al } \\
\text { descubierto }\end{array}$ & $\begin{array}{l}\text { Las profecías del cambio climático, fin del } \\
\text { mundo, CERN }\end{array}$ & $16 / 08 / 2015$ \\
\hline 37 & $\begin{array}{l}\text { Happy learning } \\
\text { español }\end{array}$ & $\begin{array}{l}\text { El cambio climático / Videos educativos para } \\
\text { niños }\end{array}$ & $31 / 01 / 2017$ \\
\hline 39 & Soloenmicasa & Cambio climático & $02 / 06 / 2007$ \\
\hline 44 & MisteriosOcultosTV & El timo del cambio climático & $23 / 01 / 2015$ \\
\hline
\end{tabular}

Ámbitos. Revista Internacional de Comunicación | ISSN: 1139-1979 | E-ISSN: 1988-5733, №. 46. (2019) 


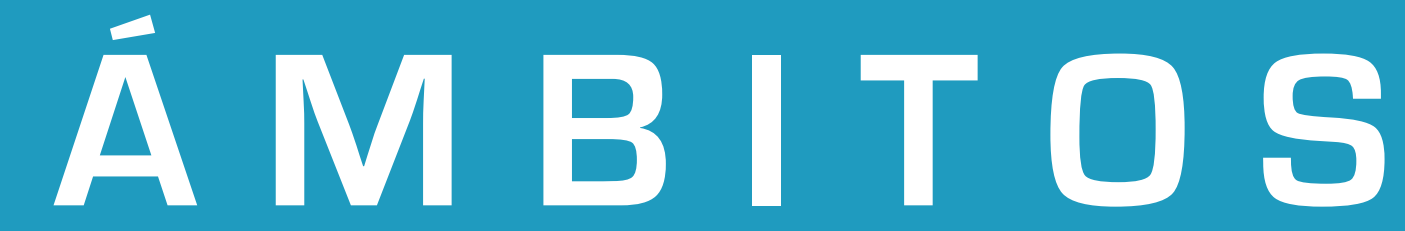

ISSN: 1139-1979 | ISSN digital: 1988-5733 | Depósito Legal: SE-1493-98

Revista Internacional de Comunicación editada por el Grupo de Investigación en Estructura, Historia y Contenidos de la Comunicación(GREHCCO) de la Universidad de Sevilla.

\section{ambitoscomunicacion@us.es}

http://institucional.us.es/ambitos

@RevistaAmbitos 